\title{
Estudio comparativo entre coronas, endocoronas y endocoronas con ausencia de una pared axial ante fuerzas de tracción.
}

\author{
Comparative study between crown, endocrown and endocrown \\ without one axial wall after tensile strength.
}

Paola Estefanía Ormaza Fonseca,* Juan Del Valle Lovato ${ }^{\ddagger}$

\section{RESUMEN}

Introducción: Las alternativas de tratamiento de órganos dentales con gran destrucción en su estructura varían, no sólo por el material de restauración, sino también por el valor económico y estético. Dentro de las alternativas existe: corona convencional, endocorona cuyo objetivo principal es la elaboración de una restauradora que evita la colocación de postes intraconducto y endocorona con ausencia de una pared axial (EPA) que se realiza cuando una pared, mesial, distal, vestibular o palatina está ausente. Objetivo: Verificar si la endocorona EPA se comporta de igual manera que las coronas convencionales y endocoronas al medir su resistencia ante fuerzas de tracción. Material y métodos: Treinta premolares fueron tratados endodóncicamente, 10 fueron preparados para recibir una corona convencional (grupo A), 10 para endocorona (grupo B) y 10 para endocorona EPA (grupo C). Se realizaron fuerzas de tracción para obtener el valor máximo en el cual las coronas fallaron. Se realizó una prueba ANOVA para comparar los resultados. Resultados: $\mathrm{Al}$ someter a los tres tipos de coronas a fuerzas de tracción los resultados obtenidos fueron: $3.04 \pm 0.55 \mathrm{MPa}$ para la corona, $7.08 \pm 1.6 \mathrm{MPa}$ para la endocorona y $6.17 \pm 1.12$ MPa para la endocorona EPA. Conclusiones: No existió diferencia significativa entre la endocorona (7.08 MPa) y la EPA (6.17 MPa), convirtiéndose en una alternativa de tratamiento con buen pronóstico en la práctica diaria.

Palabras clave: Endocorona, destrucción dentaria, diente endodóncicamente tratado, fuerzas de tracción.

\section{ABSTRACT}

Introduction: The alternatives of treatment of tooth with excessive wear vary not only by the restoration material but also by the economic and aesthetic value. Among the alternatives there is: conventional crown, endocrown whose main objective is the elaboration of a restorative that avoids the placement of intraconducting posts and endocrown without one axial wall (EPA) that is done when a wall; mesial, distal, vestibular or palatal is absent. Objective: To verify if the (EPA) behaves in the same way as the conventional crown and endocrown when measuring its resistance to tensile strength. Material and methods: 30 premolars were treated endodontically, ten were prepared to receive a conventional crown, 10 for endocrown and 10 for EPA. Tensile strength were performed to obtain the maximum value at which the crowns failed, an ANOVA test was performed to compare the results. Results: When the three types of crowns were subjected to tensile strength, the results obtained were; $3.04 \pm 0.55 \mathrm{MPa}$ for the crown, $7.08 \pm 1.6 \mathrm{MPa}$ for the endocrown and 6.17 $\pm 1.12 \mathrm{MPa}$ for the EPA endocrown. Conclusions: There was no significant difference between the endocrown (7.08 MPa) and EPA endocrown (6.17 MPa) becoming an alternative treatment with good prognosis in daily practice.

Keywords: Endocrown, excessive tooth wear, endodontically treated teeth, tensile strength.

\footnotetext{
* Odontóloga.
}

‡Profesor.

Universidad Central del Ecuador. Ecuador.

Recibido: 12 de mayo de 2020. Aceptado: 18 de mayo de 2021.

Citar como: Ormaza FPE, Del Valle LJ. Estudio comparativo entre coronas, endocoronas y endocoronas con ausencia de una pared axial ante fuerzas de tracción. Rev ADM. 2021; 78 (3): 149-154. https://dx.doi.org/10.35366/100072 


\section{INTRODUCCIÓN}

$\mathrm{L}$ a mayoría de dientes individuales que necesitan - restauraciones indirectas son dientes que han sufrido gran cantidad de pérdida dental, la localización del daño se le puede denominar como periférico, ya que presenta sus paredes axiales casi destruidas en su totalidad, otra localización de destrucción es en su parte central, aunque muchas veces se encuentran destruidos en ambas localizaciones, también los dientes que han sido sometidos a tratamientos de conductos muchas de las veces necesitan restauraciones indirectas. ${ }^{1}$

Una opción de restauración indirecta es la endocorona, la cual se le coloca sobre la cámara pulpar al diente endodonciado a través de sistemas adhesivos, los cuales van a favorecer una mejor retención. ${ }^{2,3}$ El tiempo clínico y el costo es menor, debido a que no se requiere de la colocación de poste. ${ }^{4} \mathrm{Al}$ no colocar poste se evitan las fracturas radiculares, ${ }^{5}$ además las endocoronas presentan mayor resistencia a la fractura en comparación con las coronas convencionales; ${ }^{6}$ las endocoronas poseen menor número de superficies adhesivas, lo que les hace menos susceptible a la degradación de la capa híbrida, y resisten de mejor manera a las fuerzas de compresión oblicua en comparación con las coronas convencionales más poste de fibra de vidrio. ${ }^{2,7}$ Se han realizado estudios con endocoronas sin efecto férula y con efecto férula de 1-2 mm, concluyendo que las endocoronas con efecto férula tienen mayor resistencia al fallo de carga. ${ }^{8}$ Se han realizado estudios con ausencia de una pared y preparaciones supragingivales, pero estos estudios sólo se han comparado entre endocoronas de varios materiales, concluyendo que las cerámicas a base de sílice o modificadas con circonio o polímero no tienen diferencia significativa ante fuerzas de compresión tangencial. ${ }^{3}$ Chun-Li Lin realizó un modelo elemento finito para comparar el estrés entre una restauración inlay MOD, endocorona sin paredes mesial y distal y una corona convencional con poste, llegando a la conclusión de que el estrés es mucho mayor en la incrustación mientras que entre la corona convencional y la endocorona no existe diferencia; ${ }^{9}$ sin embargo, no se han realizado pruebas de tracción en coronas que presentan ausencia de una pared, tomando en cuenta que esto disminuye la capacidad de retención de las endocoronas, por lo tanto el propósito de la presente investigación es determinar si las endocoronas que son preparadas con ausencia de una pared axial EPA presentan igual o mejor fuerza de retención en comparación con las endocoronas y coronas convencionales.

Planteamiento del problema: existen varias alternativas restauradoras en piezas dentales que han sufrido gran destrucción en su estructura o piezas que han sido sometidas a tratamientos de conductos. Una de ellas son las endocoronas, pero en determinadas ocasiones se ha perdido una pared axial, por esto ha surgido la interrogante sobre la realización de endocorona EPA.

El objetivo es verificar si la endocorona preparada sobre tres paredes axiales (EPA) se comporta de igual manera que las coronas y endocoronas convencionales al medir la resistencia ante la fuerza de tracción.

\section{MATERIAL Y MÉTODOS}

Se seleccionaron 30 premolares inferiores con longitudes y características anatómicas similares, los cuales fueron extraídos por tratamientos de ortodoncia, se les realizó profilaxis con piedra pómez y copa de caucho para luego ser almacenados en una solución de agua destilada. Las 30 piezas dentales fueron divididas en tres grupos: el primero que corresponde al grupo de coronas $(A=10)$, el segundo que se designó para las endocoronas $(B=10)$ y el tercero que fue seleccionado para las endocoronas EPA $(C=10)$.
Figura 1:

\begin{abstract}
A) Parte interna de la corona convencional. B) Parte interna

de la endocorona. C) Parte interna de la endocorona EPA.
\end{abstract}
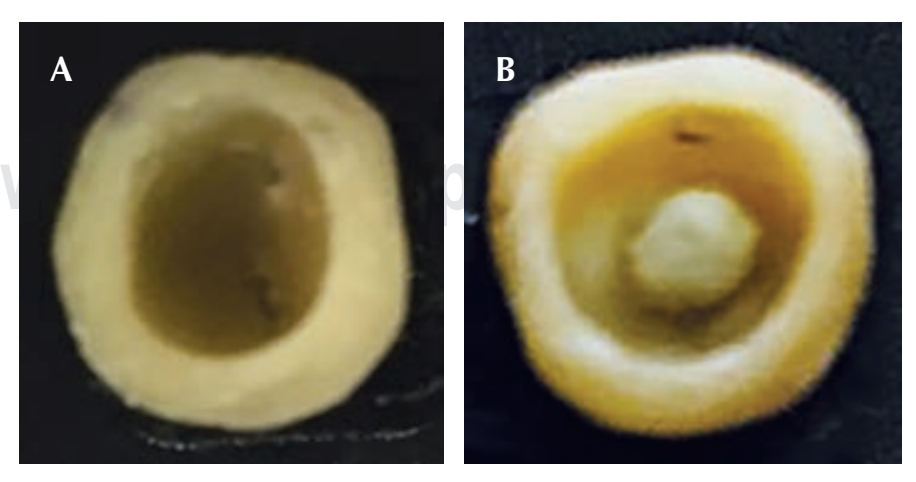
Figura 2:

Corona con gancho para realizar pruebas de tracción.

Preparación de los órganos dentales: para las coronas se usaron fresas troncocónicas de extremo redondo de $1.5 \mathrm{~mm}$, se prepararon las paredes vestibular, lingual, mesial, distal, oclusal en $1.5 \mathrm{~mm}$ con una terminación cervical en chamfer. ${ }^{10}$ Para la preparación de las endocoronas se realizó como una corona normal, además de una cavidad de $2.5 \mathrm{~mm}$ en la cámara pulpar como si los dientes hubieran sido sometidos a tratamiento de endodoncia. ${ }^{11}$ Para la variación de la endocorona EPA se realizó el mismo protocolo que la endocorona normal con la modificación que se retiró la pared distal de los órganos dentales (Figura 1).

Elaboración de las coronas: se realizó una impresión con pasta de adición pesada y liviana, se obtuvieron modelos en yeso tipo IV, se aisló el modelo y se realizaron las coronas en cerómero, además de colocar unos ganchos con alambre de ortodoncia \#7 para poder realizar las pruebas de tracción (Figura 2).

Cementación de las coronas: se realizó un microarenado de 6 segundos en cada corona de cerómero, con óxido de aluminio de $45 \mathrm{~nm}$, se lavó y secó, se colocó silano por 1 minuto hasta que éste se evaporó. Además de realizar el grabado con ácido ortofosfórico al 37\% en las 30 piezas dentales durante 15 segundos y lavar por el mismo tiempo. Se colocó adhesivo tanto en la superficie dental como en la restauración y, por consiguiente, una porción de cemento de resina dual en cada corona para proceder a la cementación, se retiraron los excesos de material y se procedió a fotopolimerizar durante 40 segundos cada cara de la pieza dental. Una vez cementadas todas las muestras, el siguiente paso fue colocarlas sobre bloques de acrílico para poder realizar la prueba a la tracción, y con la ayuda de cajas de metal preformadas con medida estándar se procedió a colocar las piezas, la altura del bloque de acrílico fue de $1.5 \mathrm{~cm}$ y el ancho de $2.5 \mathrm{~cm}$.

Pruebas de tracción: las muestras fueron sometidas a fuerzas de tracción en una máquina de test universal (Tinius Olsen) dentro del laboratorio de análisis de esfuerzos y vibraciones de la Universidad Politécnica Nacional, la fuerza se calculó en megapascales (MPa) (Figura 3).

\section{RESULTADOS}

En la estadística descriptiva (Tabla 1) se obtuvieron los siguientes resultados:

- Grupo A (corona): la muestra tiene una media de 3.04 MPa, una desviación estándar de 0.55 MPa.

- Grupo B (endocorona): la muestra tiene una media de 7.28 MPa, una desviación estándar de 1.16 MPa.

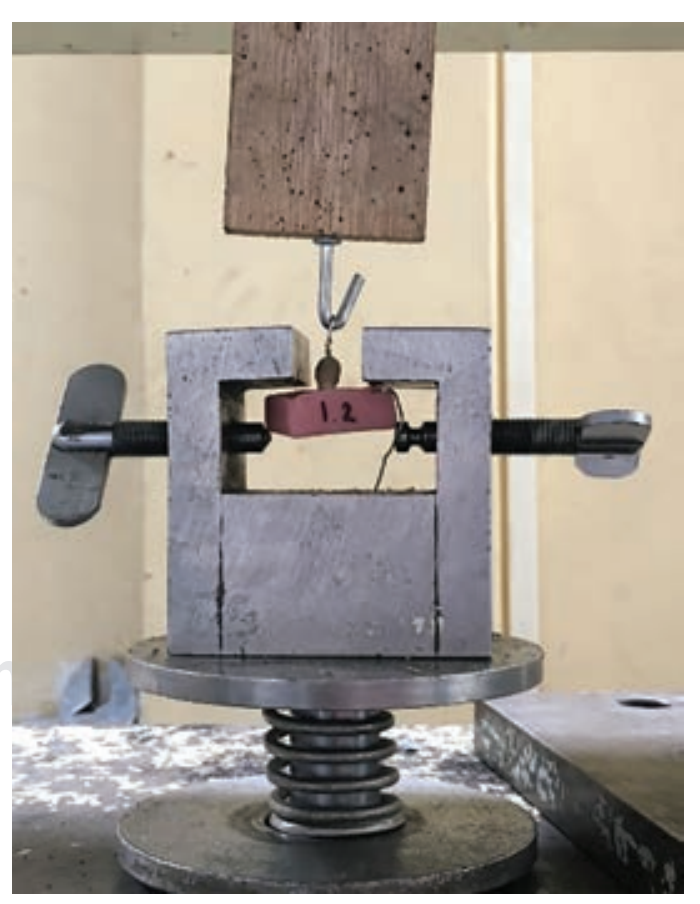

Figura 3: Prueba de tracción. 
Tabla 1: Resultados de estadística descriptiva. Resistencia de adherencia [MPa].

\begin{tabular}{|c|c|c|c|c|c|c|}
\hline \multirow[b]{3}{*}{ Grupo } & \multirow[b]{3}{*}{$\mathrm{n}$} & \multirow[b]{3}{*}{ Media \pm DE } & \multirow[b]{3}{*}{$\mathrm{EE}$} & \multicolumn{2}{|c|}{ IC 95\% para la media } & \multirow{3}{*}{$\begin{array}{c}\text { Rango } \\
\text { (mín-máx) }\end{array}$} \\
\hline & & & & \multicolumn{2}{|c|}{ Límite } & \\
\hline & & & & Inferior & Superior & \\
\hline A & 10 & $3.0470 \pm 0.55039$ & 0.17405 & 2.6533 & 3.4407 & $2.09-4.01$ \\
\hline B & 10 & $7.2870 \pm 1.15828$ & 0.36628 & 6.4584 & 8.1156 & $5.19-8.96$ \\
\hline $\mathrm{C}$ & 10 & $6.1720 \pm 1.12332$ & 0.35522 & 5.3684 & 6.9756 & $3.90-7.92$ \\
\hline Total & 30 & $5.5020 \pm 2.05761$ & 0.37567 & 4.7337 & 6.2703 & $2.09-8.96$ \\
\hline
\end{tabular}

Grupo A: corona. Grupo B: endocorona. Grupo C: endocorona EPA.

$\mathrm{DE}$ = desviación estándar; $\mathrm{EE}$ = error estándar; IC 95\% = intervalo de confianza de 95\%; mín-máx = mínimo-máximo; EPA = endocorona con ausencia de una pared axial; MPa: megapascales.

\section{Tabla 2: Prueba de normalidad Shapiro-Wilk. Resistencia de adherencia [MPa].}

\begin{tabular}{cccc} 
& & \multicolumn{2}{c}{ Shapiro-Wilk } \\
\cline { 3 - 4 } Grupo & Estadístico & gl & Sig. \\
\hline A & 0.951 & 10 & 0.684 \\
B & 0.961 & 10 & 0.798 \\
C & 0.961 & 10 & 0.794 \\
\hline
\end{tabular}

Grupo A: corona. Grupo B: endocorona. Grupo C: endocorona EPA; MPa: megapascales.

- Grupo C (endocorona EPA): la muestra tiene una media de $6.17 \mathrm{MPa}$, una desviación estándar de 1.12 MPa.

Estadística inferencial: se realizó la prueba de normalidad con la prueba de Shapiro-Wilk en la que se demostró que las muestras provienen de una población con distribución normal, ya que superan el nivel de significancia impuesto del 0.05 .

Prueba de normalidad (Tabla 2): a continuación, se determinó por medio del análisis ANOVA si las medias son o no estadísticamente iguales; primero se realizó una prueba de homogeneidad de varianzas en donde se determinó que las varianzas fueron iguales. Prueba de homogeneidad de varianzas de LEVENE.

\section{Tabla 3: Prueba de homogeneidad de varianzas de Levene. Resistencia de adherencia [MPa].}

\begin{tabular}{lccc} 
Estadístico de Levene & df1 & df2 & Sig. \\
1.786 & 2 & 27 & 0.187 \\
\hline MPa: megapascales. & & &
\end{tabular}

Tabla 4: Prueba ANOVA. Resistencia de adherencia [MPa].

\begin{tabular}{lccccc} 
& $\begin{array}{c}\text { Suma de } \\
\text { cuadrados }\end{array}$ & gl & $\begin{array}{c}\text { Media } \\
\text { cuadrática }\end{array}$ & F & Sig. \\
\hline $\begin{array}{l}\text { Entre } \\
\text { grupos }\end{array}$ & 96.622 & 2 & 48.311 & 49.867 & 0.000 \\
$\begin{array}{l}\text { Dentro de } \\
\text { grupos }\end{array}$ & 26.158 & 27 & 0.969 & & \\
Total & 122.779 & 29 & & & \\
\hline
\end{tabular}

MPa: megapascales.

La prueba de homogeneidad de varianzas de Levene se muestra en la Tabla 3. Para el criterio final de la prueba ANOVA se determinó la siguiente hipótesis:

- Ho: no existe una diferencia significativa entre las medias de la resistencia a la adherencia correspondientes a las muestras del grupo 1 , grupo 2 y grupo $3 \geq 0.05$. 
- Ha: existe una diferencia significativa entre las medias de la resistencia a la adherencia correspondientes a las muestras del grupo 1 , grupo 2 y grupo $3<0.05$.

La prueba ANOVA se muestra en la Tabla 4. Existe una diferencia significativa entre todos los grupos, para observar entre qué grupos existió diferencia significativa se realizó la prueba post hoc (prueba de Tukey). Los resultados y comparaciones de la prueba de ANOVA-post hoc-Tukey se muestran en la Tabla 5.

Se obtuvieron los siguientes resultados:

- Entre grupo A-B, $\mathrm{p}=0.000<\alpha=0.05$

- Entre grupo A-C, $p=0.000<\alpha=0.05$.

- Entre grupo B-C, $p=0.000<\alpha=0.05$.

Sí existió diferencia significativa entre todos los grupos y matemáticamente se afirma que el valor más alto es el del grupo B (endocorona).

\section{DISCUSIÓN}

El presente trabajo demostró que las endocoronas EPA tienen una mayor resistencia a la fuerza de tracción en comparación con las coronas convencionales y menor frente a las endocoronas convencionales, quizá supera a las coronas convencionales porque presenta paredes ex- ternas e internas, lo que aumente la retención, presenta menor retención en comparación con las endocoronas convencionales ya que al disminuir una pared de su estructura se elimina parte de la retención mecánica. No se han realizado estudios de tracción in vitro; sin embargo, el estudio de Otto ${ }^{12}$ realizó un seguimiento de un año a endocoronas adhesivas Cerec en molares con ausencia de dos paredes (distal y mesial), ninguna corona se fracturó ni se cementó. También en el estudio de Bindl ${ }^{13}$ se realizó un seguimiento de aproximadamente 26 meses de endocoronas adhesivas Cerec en molares, sólo una corona se salió en el mes 28. Si bien es cierto que estos estudios son clínicos, se pueden relacionar con el presente artículo, en el que los valores de retención de las endocoronas EPA tuvieron un alto valor de resistencia a la fuerza de tracción. Se recomienda realizar estudios de envejecimiento y después someterlos a compresión axial y tangencial para testear el desempeño de las endocoronas EPA, además de usar otro tipo de material para la corona.

\section{CONCLUSIONES}

Las endocoronas EPA se comportan de mejor manera que la corona normal, y con una diferencia ligeramente menor que las endocoronas convencionales al ser sometida ante fuerzas de tracción, convirtiéndose así en una alternativa de tratamiento con buen pronóstico.

Tabla 5: Prueba con ANOVA-post hoc-Tukey-comparaciones múltiples. Resistencia de adherencia [MPa].

Prueba de significancia honesta de Tukey

\begin{tabular}{|c|c|c|c|c|c|c|}
\hline \multicolumn{7}{|c|}{ Prueba de significancia honesta de Tukey } \\
\hline \multirow{2}{*}{\multicolumn{2}{|c|}{ Tipo de grupo }} & \multirow{3}{*}{$\begin{array}{c}\text { Diferencia de } \\
\text { medias (I-J) }\end{array}$} & \multirow[b]{3}{*}{$\mathrm{EE}$} & \multirow[b]{3}{*}{ Sig. } & \multirow{2}{*}{\multicolumn{2}{|c|}{$\begin{array}{c}\text { IC 95\% } \\
\text { Límite }\end{array}$}} \\
\hline & & & & & & \\
\hline \multirow[t]{2}{*}{ I } & $\mathrm{J}$ & & & & Inferior & Superior \\
\hline & B & $-4.24000^{*}$ & 0.44018 & 0.000 & -5.3314 & -3.1486 \\
\hline A & $\mathrm{C}$ & $-3.12500 *$ & 0.44018 & 0.000 & -4.2164 & -2.0336 \\
\hline \multirow[t]{2}{*}{ B } & A & $4.24000 *$ & 0.44018 & 0.000 & 3.1486 & 5.3314 \\
\hline & $\mathrm{C}$ & $1.11500 *$ & 0.44018 & 0.045 & 0.0236 & 2.2064 \\
\hline \multirow[t]{2}{*}{$\mathrm{C}$} & A & $3.12500^{*}$ & 0.44018 & 0.000 & 2.0336 & 4.2164 \\
\hline & B & $-1.11500^{*}$ & 0.44018 & 0.045 & -2.2064 & -0.0236 \\
\hline
\end{tabular}

* La diferencia de medias es significativa en el nivel 0.05. Grupo A: corona. Grupo B: endocorona. Grupo C: endocorona EPA. EE = error estándar; IC $95 \%$ = intervalo de confianza de $95 \%$. 
Al someter a los tres tipos de coronas a fuerzas de tracción, se obtuvo una resistencia en megapascales de $3.04 \pm 0.55 \mathrm{MPa}$ para la corona, $7.08 \pm 1.6 \mathrm{MPa}$ para la endocorona y $6.17 \pm 1.12 \mathrm{MPa}$ para la endocorona EPA.

\section{REFERENCIAS}

1. Zhu Z, Dong XY, He S, Pan X, Tang L. Effect of post placement on the restoration of endodontically treated teeth: a systematic review. Int J Prosthodont. 2015; 28 (5): 475-483.

2. Biacchi GR, Mello B, Basting RT. The endocrown: an alternative approach for restoring extensively damaged molars. J Esthet Restor Dent. 2013; 25 (6): 383-390.

3. Aktas G, Yerlikaya H, Akca K. Mechanical failure of endocrowns manufactured with different ceramic materials: an in vitro biomechanical study. J Prosthodont. 2018; 27 (4): 340-346.

4. Sedrez-Porto JA, Rosa WL, da Silva AF, Munchow EA, Pereira-Cenci T. Endocrown restorations: A systematic review and meta-analysis. J Dent. 2016; 52: 8-14.

5. Ramírez-Sebastia A, Bortolotto T, Cattani-Lorente M, Giner L, Roig M, Krejci I. Adhesive restoration of anterior endodontically treated teeth: influence of post length on fracture strength. Clin Oral Investig. 2014; 18 (2): 545-554.

6. Rayyan MR, Alauti RY, Abanmy MA, AlReshaid RM, Bin Ahmad $\mathrm{HA}$. Endocrowns versus post-core retained crowns for restoration of compromised mandibular molars: an in vitro study. Int J Comput Dent. 2019; 22 (1): 39-44.

7. Dietschi D, Duc O, Krejci I, Sadan A. Biomechanical considerations for the restoration of endodontically treated teeth: a systematic review of the literature--Part 1. Composition and micro- and macrostructure alterations. Quintessence Int. 2007; 38 (9): 733-743.

8. Einhorn M, DuVall N, Wajdowicz M, Brewster J, Roberts $H$. Preparation ferrule design effect on endocrown failure resistance. J Prosthodont. 2019; 28 (1): e237-e242.

9. Lin $\mathrm{CL}$, Chang $\mathrm{YH}$, Pai CA. Evaluation of failure risks in ceramic restorations for endodontically treated premolar with MOD preparation. Dent Mater. 2011; 27 (5): 431-438.

10. Shillingburg HT. Fundamentals of fixed prosthodontics. fourth edition. UK: Quintessence; 2012.

11. Guo J, Wang Z, Li X, Sun C, Gao E, Li H. A comparison of the fracture resistances of endodontically treated mandibular premolars restored with endocrowns and glass fiber post-core retained conventional crowns. J Adv Prosthodont. 2016; 8 (6): 489-493.

12. Otto T. Computer-aided direct all-ceramic crowns: preliminary 1-year results of a prospective clinical study. Int J Periodontics Restorative Dent. 2004; 24 (5): 446-455.

13. Bindl A, Mormann WH. Clinical evaluation of adhesively placed Cerec endo-crowns after 2 years--preliminary results. J Adhes Dent. 1999; 1 (3): 255-265.

Conflicto de intereses: Los autores declaran no tener ningún conflicto de intereses.

\author{
Correspondencia: \\ Juan Del Valle Lovato \\ E-mail: juanpadel_@hotmail.com
}

